\title{
O uso de ferramentas SIG para obtenção de parâmetros topográficos e de vegetação em municípios do sertão pernambucano
}

\author{
The use of SIG tools to obtain topographic and vegetation parameters in cities in \\ the Pernambuco's "sertão"
}

\author{
NORBERTO, Alison de Souza. Mestrado/Engenharia Civil \\ Universidade Federal de Pernambuco - Campus Recife. Av. Prof. Moraes Rego, 1235 - Cidade Universitária, Recife \\ - PE - Brasil. CEP: 50670-901. Telefone: (81) 99890.8140 / E-mail: alison_norberto@hotmail.com
}

\section{NASCIMENTO, Anderson Viana do. Mestrado/Engenharia Civil}

Universidade Federal de Pernambuco - Campus Recife. Av. Prof. Moraes Rego, 1235 - Cidade Universitária, Recife - PE - Brasil. CEP: 50670-901. Telefone: (81) 99500.8725 / E-mail: andersonvnascimento@outlook.com

\section{MEDEIROS, Rafaella de Moura. Graduação/Engenharia Civil}

Universidade Federal de Pernambuco - Campus Recife. Av. Prof. Moraes Rego, 1235 - Cidade Universitária, Recife - PE - Brasil. CEP: 50670-901. Telefone: (81) 99747.1160 / E-mail: rafaellamouraa1@gmail.com

\author{
ALVES, Adriana Thays Araújo. Mestrado/Engenharia Civil \\ Universidade Federal de Pernambuco - Campus Acadêmico do Agreste. Avenida Marielle Franco, s/n, km 59, \\ Caruaru - PE - Brasil. CEP: 55014-900. Telefone: (81) 99747.1160 / E-mail: adrianathaaraujo@gmail.com
}

\section{BARROS, Vitor Hugo de Oliveira. Mestrado/Engenharia Civil}

Instituto Federal do Sertão Pernambucano - Campus Serra Talhada. Rodovia PE 320, s/n - km 126, Zona Rural, Serra Talhada - PE - Brasil. Caixa Postal 78 / Telefone: (81) 99280.5562 / E-mail: vitor.barros@ifsertao-pe.edu.br

\section{RESUMO}

A demanda de informações para subsidiar estudos nas mais diversas áreas da pesquisa ambiental tem aumentado significativamente. Dentre essas informações, aquelas relativas à representação do relevo com técnicas de sensoriamento remoto e geoprocessamento são bastante eficazes na gestão ambiental, além disso, dados obtidos com essas técnicas são fundamentais em diversos estudos, possibilitando análises que subsidiam o processo de planejamento e gestão urbana. Com esse panorama, o presente trabalho objetivou aplicar o geoprocessamento utilizando o software QGIS para obtenção de informações topográficas e de cobertura vegetal (curvas de nível, relevo, declividade e NDVI) de uma região do semiárido brasileiro através de imagens do satélite LANDSAT 5 . 0 estudo é voltado para os municípios Pernambucanos de Ouricuri, Parnamirim, Santa Cruz e Terra Nova, que estão localizados no sertão do estado e no semiárido brasileiro. Os resultados mostraram a grande capacidade dessa metodologia para a obtenção desses parâmetros. O NDVI indicou uma tendência à desertificação da área de estudo.

Palavras-chave: Semiárido; Geoprocessamento; NDVI; QGIS.

\begin{abstract}
The demand for information to support studies in the most diverse areas of environmental research has increased significantly. Among this information, those related to the representation of relief with remote sensing and geoprocessing techniques are quite effective in environmental management, in addition, data obtained with these techniques are fundamental in several studies, enabling analyzes that support the urban planning and management process. With this panorama in mind, the present work aimed to apply geoprocessing using the QGIS software to obtain topographic information and vegetation cover (contour, relief, slope and NDVI) of a region in the Brazilian semiarid region using LANDSAT 5 satellite images. The study is aimed at the cities of Pernambuco in Ouricuri, Parnamirim, Santa Cruz and Terra Nova, which are located in the interior of the state and in the Brazilian semiarid region. The results showed the great capacity of this methodology to obtain these parameters. NDVI indicated a trend towards desertification of the study area.
\end{abstract}

keywords: Semiarid; Geoprocessing; NVDI; QGIS. 
NORBERTO, AS; NASCIMENTO, AV; MEDEIROS, RM; ALVES, ATA; BARROS, VHO

$O$ uso de ferramentas SIG para obtenção de parâmetros em municípios do sertão pernambucano

\section{Introdução}

Em algumas áreas da região Nordeste do Brasil têm ocorrido mudanças ambientais decorrentes da substituição da vegetação nativa pela agricultura irrigada, sendo estas raramente consideradas no manejo dos recursos hídricos (Gordon et al., 2005). Um problema já constatado em algumas microrregiões do Nordeste como, por exemplo, nas áreas irrigadas do Projeto Senador Nilo Coelho em Petrolina - Pernambuco, é o uso não sustentável dos recursos hídricos que abastecem tais áreas (Moura, 2001).

Com esse respaldo, têm-se que dados obtidos por sensoriamento remoto são fundamentais, possibilitando análises que subsidiam o processo de planejamento e gestão urbana. À essas ferramentas que auxiliam na obtenção dos dados através de sensoriamento remoto dá-se o nome de ferramentas SIG (Sistema de Informação Geográfica).

Um dos possíveis dados que podem ser obtidos através dessas ferramentas são os topográficos, que são de grande auxílio em obras de engenharia. Os principais atributos topográficos utilizados são: a elevação (curvas de nível), a declividade, a orientação e a curvatura da superfície terrestre (Ippoliti et al., 2005). Esses atributos podem ser utilizados em estudos preliminares para analisar a viabilidade da uma barragem, o traçado de uma rodovia/ferrovia, áreas de jazidas e bota-fora, dentre outros.

Existem diversas técnicas de processamento de imagens que aperfeiçoam a exploração dos dados de sensores remotos, tais como as que se referem aos índices de vegetação. Melo et al. (2011) afirmam que analisar a densidade e a espacialidade da cobertura vegetal torna-se um importante mecanismo para estudos voltados para análise da degradação da paisagem, gestão e planejamento dos recursos naturais, compreensão dos processos hidrológicos, diagnóstico do dinamismo no espaço agrário, entre outras finalidades.

Um índice muito utilizado nestas análises é o Índice de Vegetação de Diferença Normalizada (NDVI), que é um indicador numérico chamado também de índice biofísico, que auxilia no entendimento da dinâmica da vegetação. É um parâmetro com medidas radiométricas que relacionam a abundância relativa através da atividade da vegetação verde, teor de clorofila, índice de área foliar e biomassa verde (Jensen, 2009; Galvanin et al., 2014). É calculado utilizando as porções da energia eletromagnética refletida pela vegetação nas bandas do vermelho (comprimento de onda $=0,6$ micrômetros) e do Infravermelho próximo (comprimento de onda = 0,8 micrômetros).

Esse parâmetro é muito utilizado na avaliação da espacialização e da evolução da cobertura vegetal (Bilar et al., 2018). Sendo, portanto, útil para monitoramento de áreas de preservação ambiental (Gamarra et al., 2016) e para análise de degradação ambiental de bacias hidrográficas (Melo et al., 2011). Dessa forma, os índices de vegetação são utilizados para inferir e monitorar a cobertura e densidade da vegetação através de imagens de sensores multiespectrais, pois eles permitem a estimativa da biomassa ou do índice de área foliar (IAF) (Parise e Vettorazzi, 2005). É possível, ainda, avaliar a mudança temporal da cobertura vegetal e possíveis impactos na região (Santos et al., 2018). Focando em projetos de drenagem e sustentabilidade de cidades, as áreas com cobertura vegetal são um ótimo indicador, pois são áreas permeáveis, contribuem para a regularização do microclima urbano e podem constituir espaços de lazer (Fedorov e Fonseca, 2002). 
NORBERTO, AS; NASCIMENTO, AV; MEDEIROS, RM; ALVES, ATA; BARROS, VHO

$O$ uso de ferramentas SIG para obtenção de parâmetros em municípios do sertão pernambucano

$\mathrm{Na}$ literatura nacional diversos estudos são encontrados utilizando NDVI para análises ambientais em diversas localidades: Reservatório de Sobradinho - BA (Azevedo et al., 2018), Parque Estadual Mata da Pimenteira, Serra Talhada - PE (Bilar et al., 2018), planície costeira do estado do Piauí (Sousa et al., 2016), reservatório de Itaparica - PE (Matos et al., 2015), Pantanal de Cáceres MT (Galvanin et al., 2014), microbacia hidrográfica do Riacho dos Cavalos, Crateús - CE (Melo et al., 2011), Fazenda Morro Grande, Araras - SP (Parise e Vettorazzi, 2005).

Assim, o presente trabalho tem como objetivo principal utilizar ferramentas SIG para a obtenção de informações topográficas e de cobertura vegetal em municípios Pernambucanos de Ouricuri, Santa Cruz, Parnamirim e Terra Nova por meio de geoprocessamento no software QGIS.

\section{Material e métodos}

Os munícipios estudados estão localizados no Estado do Pernambuco e fazem parte das Mesorregiões de Sertão (Ouricuri, Parnamirim e Santa Cruz) e São Francisco (Terra Nova).

A vegetação é composta predominante por Caatinga Hipoxerófila. Os municípios são caracterizados como uma unidade ambiental da depressão sertaneja, com relevo suave a ondulado (Damasceno, 2007). O clima é de semiárido (Embrapa, 2020a). Os verões são quentes e úmidos e quase toda precipitação anual ocorre nesse período. Os invernos são mornos e secos, com diminuição de chuvas; as mínimas podem chegar a $15^{\circ} \mathrm{C}$. As primaveras são muito quentes e secas, com temperaturas muito altas, que em que algumas ocasiões podem chegar a mais de $40^{\circ} \mathrm{C}$.

Quanto à hidrografia, Ouricuri está inserido na bacia hidrográfica do Rio Brígida, Terra Nova está inserido na bacia do Rio Terra Nova (APAC, 2020b; 2020c). Por sua vez, Santa Cruz está nos domínios das bacias hidrográficas dos rios das Garças e Brígida (APAC, 2020a; 2020b).

Pode-se observar na Figura 1 a posição geográfica da região de estudo.

Figura 1 - Localização da área de estudo no mapa do Brasil

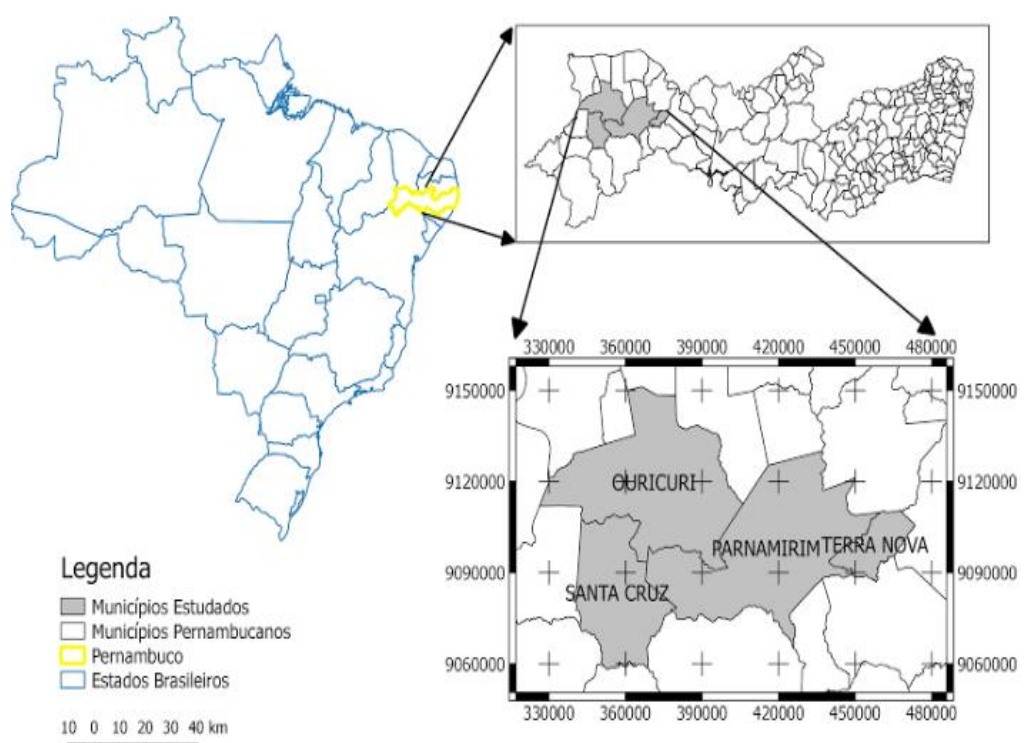

Fonte: Pesquisa direta. 
NORBERTO, AS; NASCIMENTO, AV; MEDEIROS, RM; ALVES, ATA; BARROS, VHO

$O$ uso de ferramentas SIG para obtenção de parâmetros em municípios do sertão pernambucano

Dessas três bacias hidrográficas, Brígida, Garças e Terra Nova, a Bacia do Rio Brígida é a mais importante em extensão, abrangendo uma área de $13.495,73 \mathrm{~km}^{2}$ que correspondente a $13,73 \% \mathrm{da}$ superfície total do estado de Pernambuco. Está localizada no alto Sertão de Pernambuco com coordenadas entre $07^{\circ} 19^{\prime} 02^{\prime \prime}$ e $08^{\circ} 36^{\prime} 32^{\prime \prime}$ de latitude sul e $39^{\circ} 17^{\prime} 33^{\prime \prime}$ e $40^{\circ} 43^{\prime} 06^{\prime \prime}$ de longitude oeste. Como fronteiras, a referida bacia limita-se ao norte com os Estados do Ceará e Piauí e com o grupo de bacias de pequenos rios interiores 9 - GI9 (UP28), ao sul com a bacia do riacho das Garças (UP12) e com o grupo de bacias de pequenos rios interiores 6 - GI6 (UP25), a leste com a bacia do rio Terra Nova (UP10) e o grupo de bacias de pequenos rios interiores 5 - GI5 (UP24), e a oeste com o Estado do Piauí. Na Figura 2 é apresentada a delimitação das Bacias hidrográficas de Pernambuco.

Figura 2 - Delimitação das bacias hidrográficas de Pernambuco

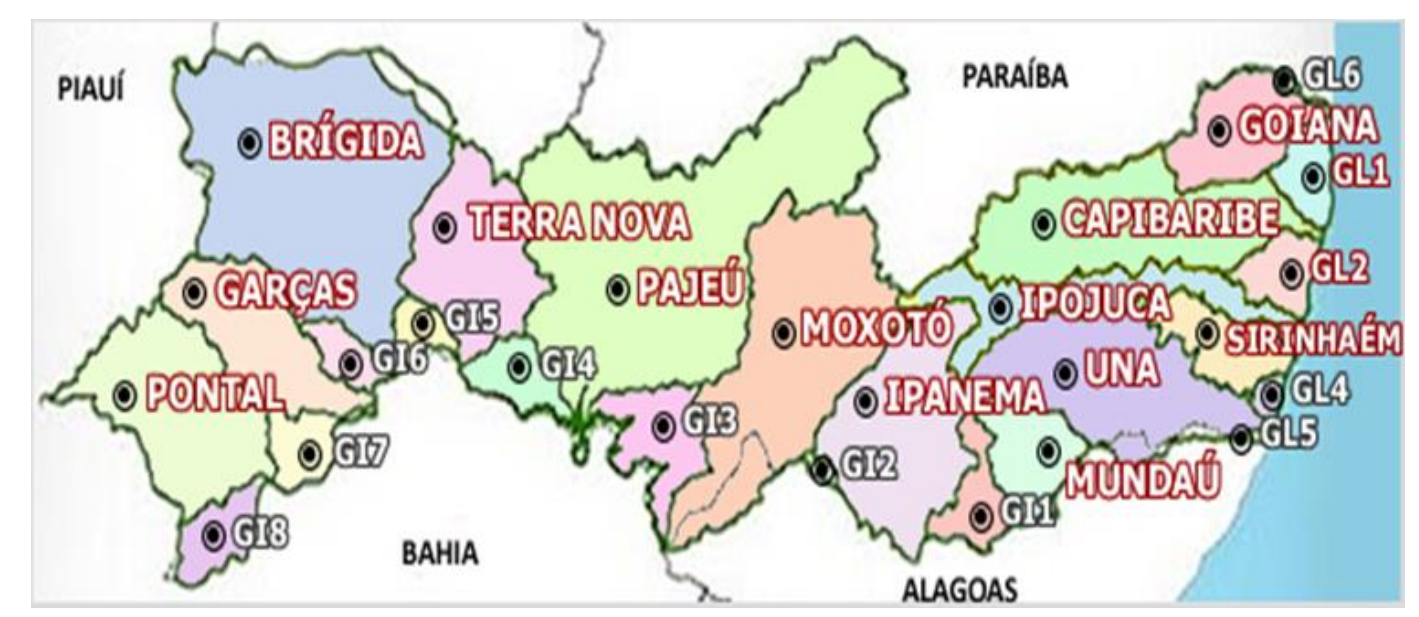

Fonte: APAC, 2012.

No presente trabalho, analisa-se a área de estudo com o auxílio do sensoriamento remoto utilizando uma ferramenta de SIG, o QGIS, que é um software gratuito que permite a criação de mapas em diferentes formatos e projeções. As análises realizadas são: análise da elevação do terreno com curvas de nível pelo Modelo Digital de Elevação, avaliação do relevo sombreado, análise da declividade do terreno em graus e em percentual e cálculo do índice de vegetação da área (NDVI).

Para realizar a análise de vegetação são utilizadas imagens obtidas pelo LandSat 5 , que possuem uma precisão (resolução do pixel) de $30 \mathrm{~m}$, na órbita 217 com ponto 66, órbita 217 com ponto 65 e na órbita 216 com ponto 66 , com data de 30 de julho de 2006. Essas imagens são fornecidas pelo o Instituto Nacional de Pesquisas Espaciais (INPE). Foi realizado o mosaico das imagens por bandas, de forma a abranger toda a área de estudo. Para a construção do mapa de índice de vegetação são utilizadas as bandas 3 (vermelho) e 4 (infravermelho) de cada imagem no processo, mas não empilhadas e sim separadamente.

Para a elaboração do modelo de elevação digital usado nos mapas de curva de nível, relevo sombreado e de declividades, são utilizadas imagens SRTM obtidas através do site da Empresa Brasileira de Pesquisa Agropecuária (EMBRAPA). Vale salientar que todas as imagens utilizadas neste trabalho foram convertidas para o datum SIRGAS 2000 fuso 24S, para compatibilizar com as outras informações do projeto. 
NORBERTO, AS; NASCIMENTO, AV; MEDEIROS, RM; ALVES, ATA; BARROS, VHO

$O$ uso de ferramentas SIG para obtenção de parâmetros em municípios do sertão pernambucano

Para análise do relevo são utilizadas as imagens do LANDSAT fornecidas pela EMBRAPA, o qual apresenta uma inclinação da superfície representada. As quatro imagens são nomeadas no site como SB-24-Y-C, SB-24-Y-D, SC-24-V-A e SC-24-V-B.

Para a definição das curvas de nível é utilizada a ferramenta de extração de contorno localizado no Menu Raster da barra de ferramentas. Para a elaboração do mapa de sombreamento e declividade em graus é necessário acessar o submenu Análise do Terreno, dentro do Menu Raster. Vale salientar que na elaboração do mapa de sombreamento é utilizado azimute de $315^{\circ}$ e ângulo vertical de $45^{\circ}$. No caso da elaboração da declividade em percentual é utilizada a ferramenta Slope, que está localizada dentro da caixa de ferramentas de processamento.

Por fim, é feita análise de índice de vegetação, que é feita pela ferramenta Vegetation Index (slope based), que se utiliza das bandas 3 e 4 das imagens do INPE separadamente para realizar o cálculo do NDVI. Por meio da criação de shapefiles com o uso das imagens fornecidas pelo INPE são feitas as delimitações de algumas das estradas da área, alguns corpos hídricos (reservatórios) e das áreas urbanas.

\section{Resultados e discussão}

Na Figura 3 é mostrada uma representação matemática computacional da distribuição do relevo na superfície da área de estudo. As curvas de nível mostradas foram obtidas a cada $20 \mathrm{~m}$.

Figura 3 - Curvas de nível

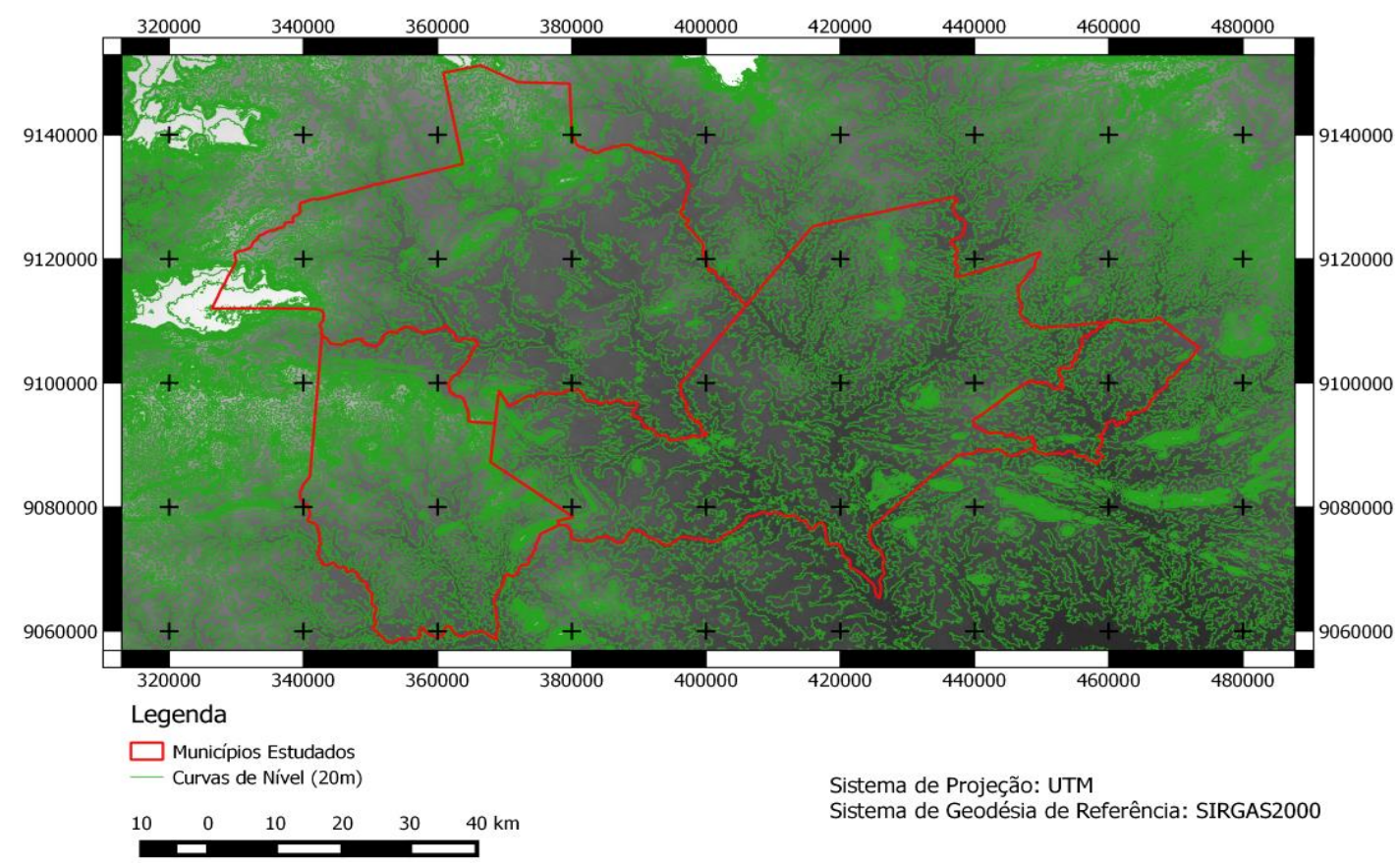

Fonte: Pesquisa direta.

Deve-se notar que, devido à resolução das imagens de satélite, que no caso é de $30 \mathrm{~m}$, as curvas de nível com precisão de $20 \mathrm{~m}$ podem apresentar um desvio em relação à realidade. Entretanto, 
NORBERTO, AS; NASCIMENTO, AV; MEDEIROS, RM; ALVES, ATA; BARROS, VHO

$O$ uso de ferramentas SIG para obtenção de parâmetros em municípios do sertão pernambucano

esses dados são importantes para um estudo inicial de locação de uma rodovia ou de uma barragem, por exemplo.

Na Figura 4 é mostrada a representação do mapa da área de estudo com relevo sombreado.

Figura 4 - Relevo sombreado

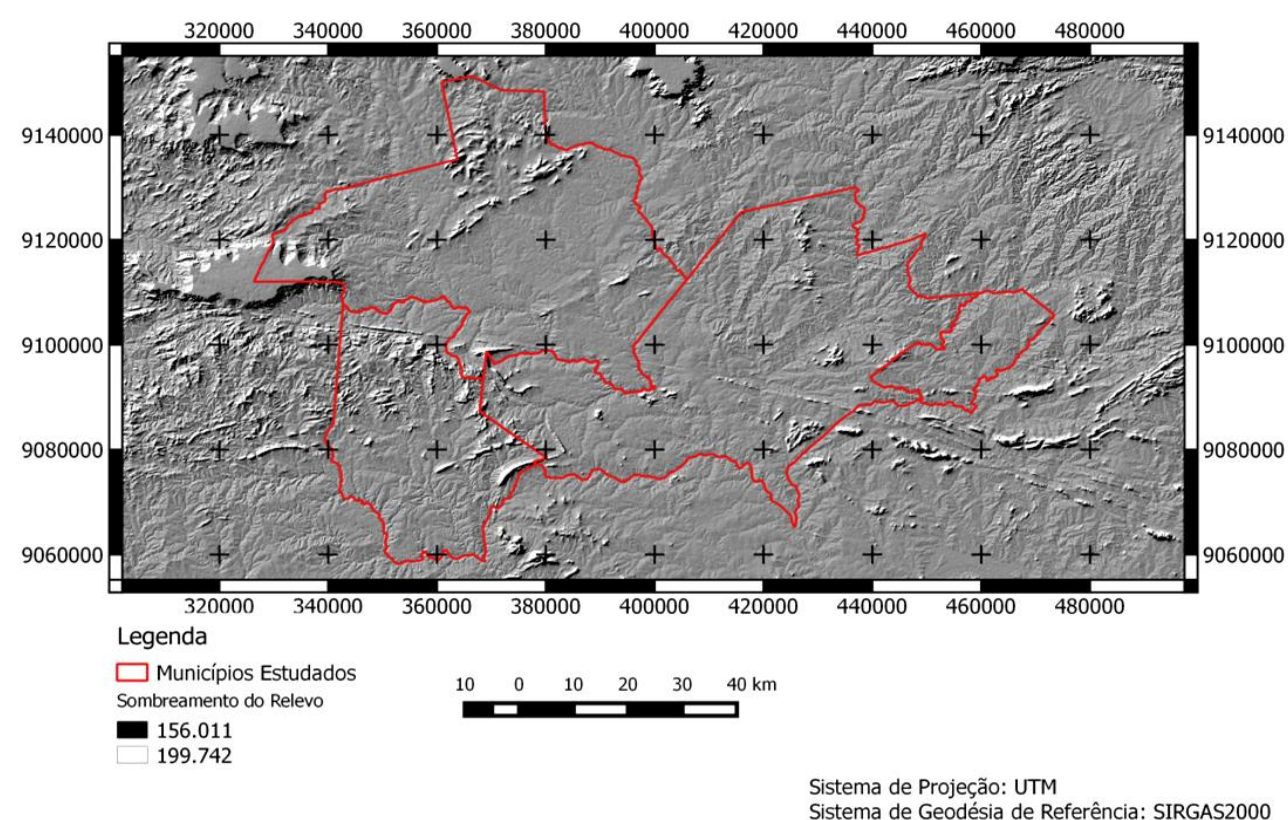

Fonte: Pesquisa direta.

Analisando a imagem, fica claro que a região de estudo apresenta uma certa homogeneidade no formato do relevo, com algumas elevações em pequenas áreas.

É possível notar que a área avaliada em sua maior extensão não possui grandes depressões, aspectos bem comuns da região. 0 perfil do relevo obtido através dessa técnica de geoprocessamento, por si só, não é muito relevante para uma análise muito complexa de um problema de engenharia. Porém, pode ser uma ótima ferramenta para a tomada de decisão em relação à problemas de fragilidade ambiental, erosão de solo, entre outros (Souza et al., 2011).

$\mathrm{Na}$ Figura 5 é apresentada a carta de declividade da área de estudo em porcentagem. As áreas de tonalidades mais escuras são indicativas de maior declividade, enquanto as áreas com tons mais claros representam uma menor declividade. Nota-se que existe uma quantidade maior de áreas com uma baixa declividade. Também é possível notar que o município de Santa Cruz é o que possui uma maior área com zonas de maior altitude. 
NORBERTO, AS; NASCIMENTO, AV; MEDEIROS, RM; ALVES, ATA; BARROS, VHO

$O$ uso de ferramentas SIG para obtenção de parâmetros em municípios do sertão pernambucano

Figura 5 - Declividade em percentual da área estudada

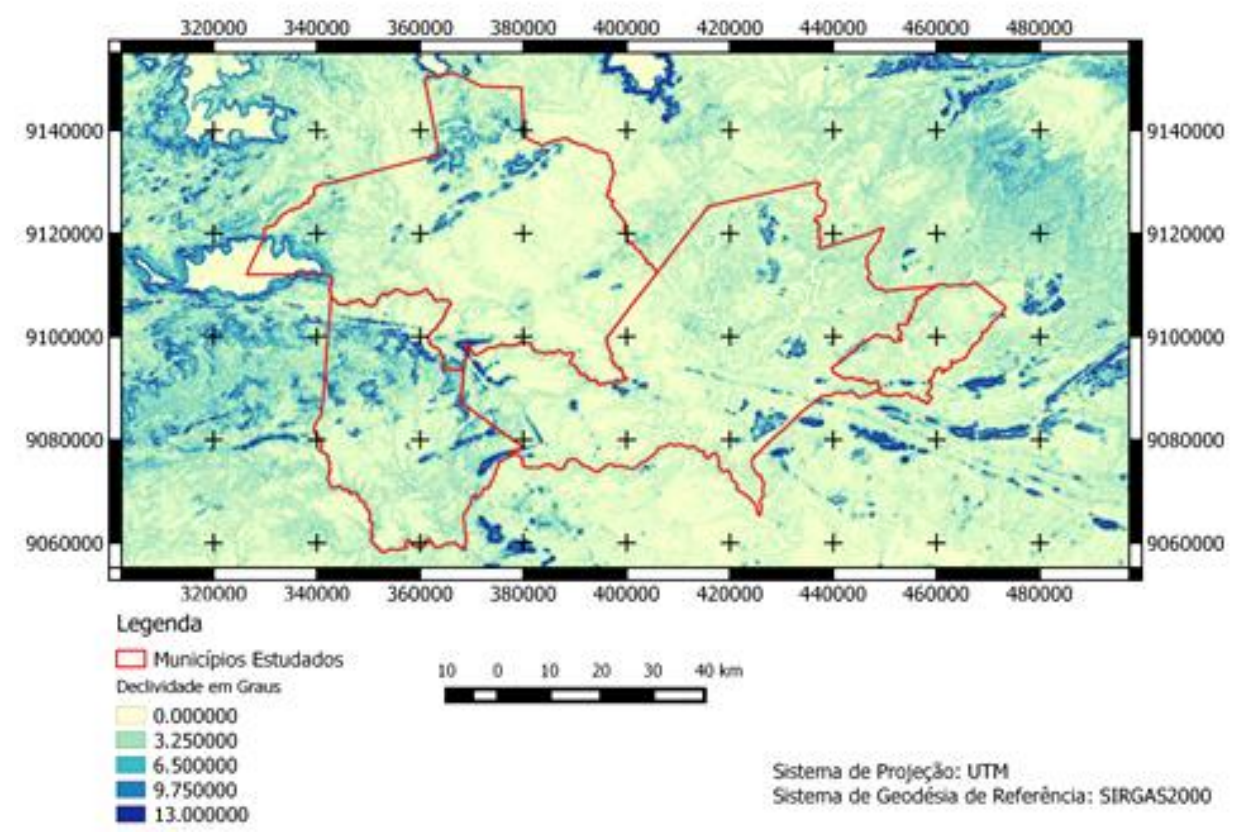

Fonte: Pesquisa direta.

Em relação às classes de declividade, segundo Lepsch et al. (1991) pode-se afirmar que as regiões mais escuras do mapa são classificadas como de relevo forte ondulado (12\%-20\%). A maior área está entre $0 \%$ e 3,25\%, sendo classificado como relevo plano (0\%-3\%). Essa classificação também foi adotada por Corseuil e Campos (2007). Na Figura 6 é apresentado um mapa da área de estudo contendo o NDVI.

Figura 6 - Índice de Vegetação por Diferença Normalizada (NDVI) da área estudada

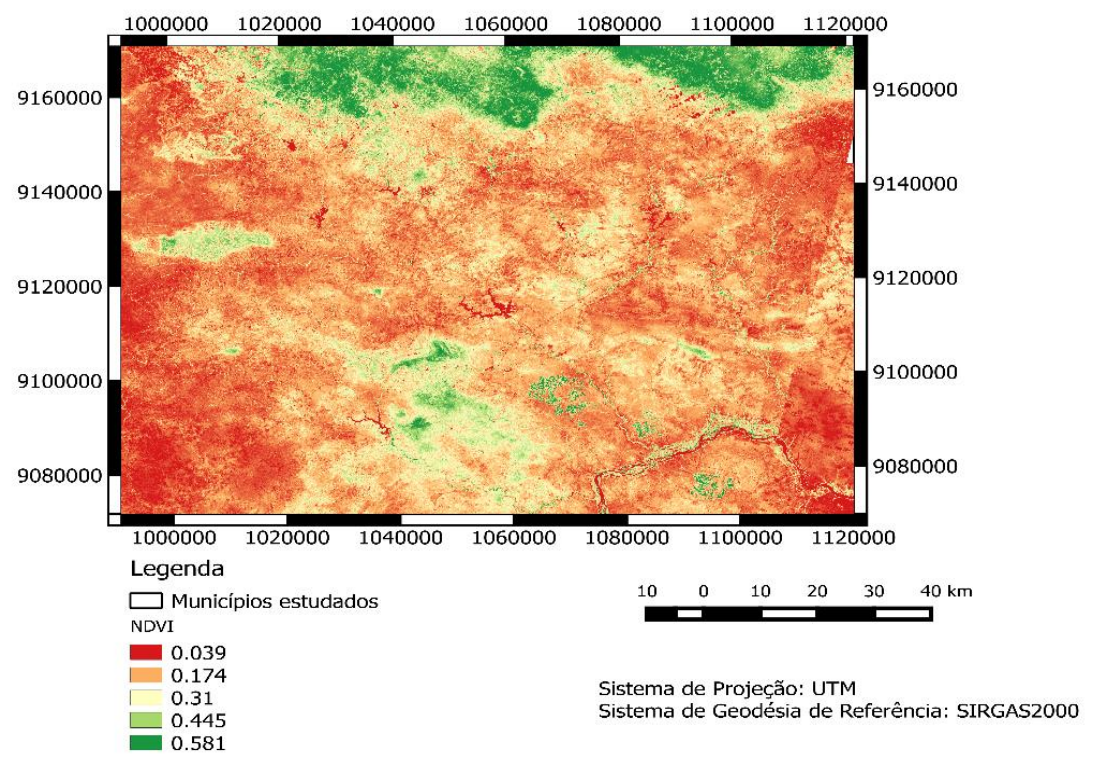

Fonte: Pesquisa direta. 
NORBERTO, AS; NASCIMENTO, AV; MEDEIROS, RM; ALVES, ATA; BARROS, VHO

$O$ uso de ferramentas SIG para obtenção de parâmetros em municípios do sertão pernambucano

O NDVI é um indicador cujo valor varia entre -1 e +1 (Sousa et al., 2016; Matos et al., 2015), de modo que quanto mais próximo de 1 maior é a presença de vegetação e quanto mais próximo de 1, maior é a presença de solo exposto (Sousa et al., 2016). Assim, através do mapa do NDVI mostrado na Figura 6, é possível afirmar que a área possuía um baixo índice de áreas verdes no período que foram formuladas as imagens de satélite, situação bastante comum em regiões do semiárido nordestino, por se tratar de uma região de clima predominante seco. Deve-se destacar que o sertão pernambucano possui um maior índice de precipitação no verão/outono, ou seja, justamente nos meses anteriores ao mês de obtenção da imagem (julho), além disso esse regime não aparenta ser influenciado por fenômenos climáticos (El Niño e La Niña), o que reforça mais ainda a validade do resultado obtido por esse método (Fechine e Galvíncio, 2009; Nóbrega et al., 2015).

Considerando o regime de chuva dos meses anteriores ao estudado, segundo o monitoramento pluviométrico fornecido pela APAC (APAC, 2020d), a cidade com maior média mensal entre as escolhidas foi Ouricuri, com média de $46,8 \mathrm{~mm}$, sendo consideravelmente maior do que a série histórica dos anos anteriores para esses meses no município, que gira em torno de $15 \mathrm{~mm}$.

Assim, pode-se concluir que o resultado obtido no mapa era o esperado, pois se trata de uma região com baixo índice de vegetação, com predominância da caatinga, tipo de mata com baixo índice folicular. Outro aspecto ponderador é ascensão e crescimento dos centros urbanos.

Na sequência, na Figura 7 são mostrados os resultados da delimitação dos corpos hídricos, das mais importantes rodovias da área de estudo e das áreas urbanas que eram visualizáveis na imagem fornecida pelo INPE, para as quatro cidades avaliadas.

Figura 7 - Delimitação de corpos hídricos, estradas e centros urbanos.

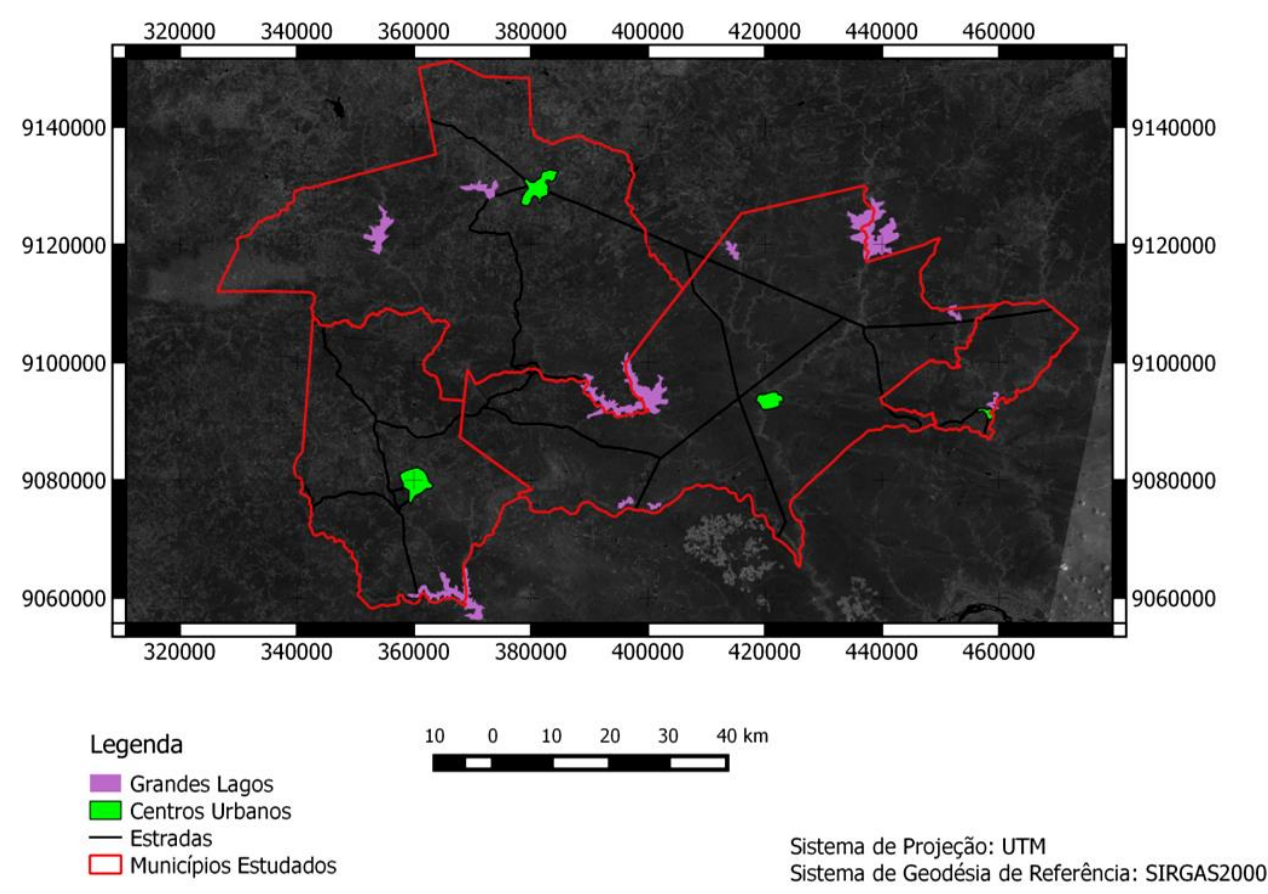

Fonte: Pesquisa direta. 
NORBERTO, AS; NASCIMENTO, AV; MEDEIROS, RM; ALVES, ATA; BARROS, VHO

$O$ uso de ferramentas SIG para obtenção de parâmetros em municípios do sertão pernambucano

É possível verificar a ocorrência de cursos de água na região, sobretudo caracterizados como grandes lagos, basta apenas observar os menores índices obtidos na avaliação do NDVI. Avaliando em conjunto com o mapa do NDVI, também é possível afirmar que a baixa densidade de vegetação influencia diretamente na capacidade manutenção do volume dos corpos hídricos.

Devido a todos os fatores e resultados apresentados, pode-se afirmar que a área estudada possui uma tendência de desertificação, pois apresenta pequenas corpos hídricos, grandes áreas planas e o clima semiárido. Para avaliar com mais precisão o risco de desertificação seriam necessários estudos mais aprofundados das características dos solos, em diferentes datas com regimes pluviométricos distintos.

\section{Conclusões}

O uso das ferramentas conhecidas do sensoriamento remoto como auxílio à avaliação de parâmetros de relevo e vegetação já se provaram de grande importância em diferentes tipos de estudos que concernem o ambiente natural, especialmente os recursos hídricos e vegetais.

Demonstrou-se que o NDVI pode ser um aliado na avaliação dos parâmetros físicos das bacias hidrográficas, a partir do momento em que a cobertura vegetal representa um componente importante da regulação do clima e dos parâmetros hidrológicos.

Os dados apresentados no presente estudo podem ser indicativos de um possível processo de desertificação da região, tema que merece uma abordagem com mais ênfase e mais aprofundada, já que outras localidades do semiárido também apresentam tal indicação. Para uma análise mais acertada dessa questão são necessários mais estudos tanto espaciais quanto temporais.

Logo, percebe-se que as técnicas de sensoriamento remoto e geoprocessamento são bastante eficazes no que toca a análise das condições naturais, e que por meio delas é possível detectar áreas sob vulnerabilidade natural para melhor planejamento do uso dos recursos destinados ao combate à degradação do solo.

Por fim, fica claro, através dos resultados apresentados, o potencial dos dados obtidos através do processamento de imagens de satélites em auxiliar na tomada de decisões em diversas áreas do conhecimento. Reforça-se, assim, o caráter preliminar da presente pesquisa, sendo parte de um projeto maior da análise em períodos distintos através das mesmas ferramentas e da aplicação prática em outras pesquisas.

\section{Referências}

AGÊNCIA PERNAMBUCANA DE ÁGUAS E CLIMAS - APAC. Bacias hidrográficas - Bacia do Riacho das Garças. Disponível em: http://www.apac.pe.gov.br/pagina.php?page_id=5\&subpage_id=24. Acessado em: 20 mar. 2020 (a). 
NORBERTO, AS; NASCIMENTO, AV; MEDEIROS, RM; ALVES, ATA; BARROS, VHO

$O$ uso de ferramentas SIG para obtenção de parâmetros em municípios do sertão pernambucano

AGÊNCIA PERNAMBUCANA DE ÁGUAS E CLIMAS - APAC. Bacias hidrográficas - Bacia do Rio Brígida. 2012. Disponível em <http://www.apac.pe.gov.br/pagina.php?page_id=5\&subpage_id=13>. Acessado em 15 de março de 2020 (b).

AGÊNCIA PERNAMBUCANA DE ÁGUAS E CLIMAS - APAC. Bacias hidrográficas - Bacia do Rio Terra Nova. Disponível em: http://www.apac.pe.gov.br/pagina.php?page_id=5\&subpage_id=22. Acessado em: 20 mar. 2020 (c).

AGÊNCIA PERNAMBUCANA DE ÁGUAS E CLIMAS - APAC. Monitoramento Pluviométrico. Disponível em: http://www.apac.pe.gov.br/meteorologia/monitoramento-pluvio.php. Acessado em: 28 abr. 2020 (d).

AZEVEDO, S.C.; CARDIM, G.P.; PUGA, F.; SINGH, R.P.; SILVA, E.A. Analysis of the 2012-2016 drought in the northeast Brazil and its impacts on the Sobradinho water reservoir. Remote Sensing Letters, 9:439447, 2018.

BILAR, A. B. C.; PIMENTEL, R. M. M.; CERQUEIRA, M. A. Monitoramento da cobertura vegetal através de índices biofísicos e gestão de áreas protegidas. Geosul, 33:236-259, 2018.

CORSEUIL, C. W.; CAMPOS, S. Geoprocessamento aplicado na determinação das classes de declive e de uso das terras da microbacia do Arroio Ajuricaba - Marechal Cândido Rondon - PR. Revista Energia Agrícola, 22:33-41, 2007.

DAMASCENO, M. M. Composição bromatológica de forragem de espécies arbóreas da Caatinga Paraibana em diferentes altitudes. Dissertação - Mestrado em Zootecnia - Sistemas Agrosilvipastoris no Semiárido. Patos, PB: UFCG, 60 p., 2007.

EMPRESA BRASILEIRA DE PESQUISA AGROPECUÁRIA - EMBRAPA. Aspectos Ambientais. Disponível em: https://www.agencia.cnptia.embrapa.br/gestor/territorio_sisal/arvore/CONT000fckg3dhc02wx5eo0 a2ndxyp208858.html. Acesso em: 20 mar. 2020 (a).

EMPRESA BRASILEIRA DE PESQUISA AGROPECUÁRIA - EMBRAPA. Relevo do Brasil. Disponível em: < http://www.relevobr.cnpm.embrapa.br/>. Acessado em 15 de março de 2020 (b).

FECHINE, J. A. L.; GALVÍNCIO, J. D. Tendência das precipitações na Bacia Hidrográfica do Rio Brígida - Estado de Pernambuco. Revista Brasileira de Geografia Física, 2:01-10, 2009.

FEDOROV, D.; FONSECA, L. M. G. Restau: image restoration 0.0. 15. São José dos Campos: INPE, Divisão de Processamento de Imagens, 2002. 
NORBERTO, AS; NASCIMENTO, AV; MEDEIROS, RM; ALVES, ATA; BARROS, VHO

$O$ uso de ferramentas SIG para obtenção de parâmetros em municípios do sertão pernambucano

GALVANIN, E. A. S.; NEVES, S. M. A. S.; CRUZ, C. B. M.; NEVES, R. J.; JESUS, P. H. H.; KREITLOW, J. P. Avaliação dos índices de vegetação NDVI, SR e TVI na discriminação de fitofisionomias dos ambientes do Pantanal de Cáceres/MT. Ciência Florestal, Santa Maria, 24:707-715, 2014.

GAMARRA, R. M.; TEIXEIRA-GAMARRA, M. C.; CARRIJO, M. G. G.; PARANHOS FILHO, A. C. Uso do NDVI na análise da estrutura da vegetação e efetividade da proteção de Unidade de Conservação no Cerrado Revista RAEGA, 37:307-332, 2016.

GORDON, L.J.; STEFFEN, W.; JÖNSSON, B.F.; FOLKE, C.; FALKENMARK, M.; JOHANNESEN, Å. Human modification of global water vapor flows from the land surface. Proceedings of the National Academy of Sciences, 102:7612-7617, 2005.

INSTITUTO NACIONAL DE PESQUISAS ESPACIAIS - INPE. Catálogo de Imagens. Disponível: <http://www.dgi.inpe.br/CDSR/>. Acesso: 08 de julho de 2016.

IPPOLITI, G. A.; COSTA, L. M. da; SCHAEFER, C. E. G. R.; FERNANDES FILHO, E. I.; GAGGERO, M. R. Análise digital do terreno: ferramenta na identificação de pedoformas em microbacia na região de “Mar dos Morros (MG)". Revista Brasileira de Ciência do Solo, 29:269-276, 2005.

JENSEN, J. R. Sensoriamento Remoto do Ambiente: uma perspectiva em recursos terrestres, traduzida pelo Instituto Nacional de Pesquisas Espaciais - INPE. São Paulo, Parêntese, 2009.

LEPSCH, I. F.; BELLINAZZI JÚNIOR, R.; BERTOLINI, D.; ESPÍNDOLA, C. R. Manual para levantamento utilitário do meio físico e classificação de terras no sistema de capacidade de uso. Campinas, Sociedade Brasileira de Ciência do Solo, 175 p., 1991.

MATOS, R. C. M.; CANDEIAS, A. L. B.; AZEVEDO, J. R. G.; HATTERMANN, F. F.; KOCH, H.; KLEINSCHMIT, B. Análise multitemporal do albedo, NDVI e temperatura no entorno do Reservatório de Itaparica - PE: anos de 1985 e 2010. Revista Brasileira de Cartografia, 3:545-554, 2015.

MELO, E. T.; SALES, M. C. L.; OLIVEIRA, J. G. B. Aplicação do índice de vegetação por diferença normalizada (NDVI) para análise da degradação ambiental da microbacia hidrográfica do Riacho dos Cavalos, Crateús - CE. Revista RAEGA, 23:520-533, 2011.

MOURA, M. S. B. Fenologia e consumo hídrico da goiabeira (Psidium guajava L.) irrigada. Dissertação (mestrado), Universidade Federal da Paraíba, Campina Grande - PB, 124p., 2001.

NÓBREGA, R. S.; FARIAS, R. F. L.; SANTOS, C. A. C. Variabilidade temporal e espacial da precipitação pluviométrica em Pernambuco através de índices de extremos climáticos. Revista Brasileira de Meteorologia, 30:171-180, 2015. 
NORBERTO, AS; NASCIMENTO, AV; MEDEIROS, RM; ALVES, ATA; BARROS, VHO

$O$ uso de ferramentas SIG para obtenção de parâmetros em municípios do sertão pernambucano

PARISE, F.J.O.; VETTORAZZI, C.A. Análise de dados de produção em um pomar jovem de laranjeiras Hamlin: Relações com a resposta espectral. Revista Brasileira de Fruticultura, 27:43-47, 2005.

SANTOS, M. N. S.; ALMEIDA, J. A. C.; ROCHA, J. L. S.; VIDAL, M. R. Uso do NDVI para análise temporal da cobertura vegetal município de Parauapebas - PA. In: III Encontro de Pós-Graduação. Anais. p. 1-9. 2018.

SOUSA, R. S.; VALLADARES, G. S.; ESPÍNDOLA, G. M. Análise do índice de vegetação (NDVI) e vulnerabilidade ambiental da planície costeira do estado do Piauí. Revista Casa da Geografia de Sobral, 18:82-99, 2016.

SOUZA, M. M.; COSTA, L. H.; CARVALHO, D. A. S. Utilização de ferramentas de geoprocessamento para mapear as fragilidades ambientais na área de influência direta da UHE de Belo Monte, no estado do Pará. Revista Espaço Plural, 12:73-85, 2011. 